\title{
The hydrodynamic dust retention modeling process with a foam layer for open linear dynamic technological systems at construction industry enterprises
}

\author{
Vadim Bespalov ${ }^{1, *}$, Oksana Gurova ${ }^{1}$, Irina Tsarevskaya $^{1}$ and Ludmila Alekseenko ${ }^{1}$ \\ ${ }^{1}$ Don State Technical University, Rostov, Russia
}

\begin{abstract}
The physical essence of the reducing air pollution hydrodynamic method by means of the foamy method as the most effective dust retention technology for open long sources at construction industry enterprises is presented in the article. The mathematical description of this technology has been performed, taking into account the physical and chemical properties of the dust and foam bubble, as well as the parameters of the return of dust particles to technological raw material due to various physical mechanisms for their capture. The obtained parametric dependence of dust retention efficiency provides the possibility of its predicted calculation as a resultant parameter for the considering technology, taking into account the interaction of dust with a foam layer.
\end{abstract}

\section{Introduction}

At present, the operation of construction industry enterprises, for which belt conveyors are one of the main types of linear dynamic systems of process equipment, is accompanied by intensive formation and release of particles [1,2].

Analysis of modern studies results [3] allows us to assume that the most effective technology for the retention of inorganic dust PM2,5 and PM10 with a $\mathrm{SiO}_{2}>70 \%$ for belt conveyors at construction industry enterprises is the hydrodynamic method based on the foam layer application. However, the dust retention management technology which also applies to very dynamic systems is possible only on the scientific justification basis and modeling of both its individual parameters and efficiency as its resulting parameter which will ensure maximum dust concentration in the air of working zones and the surface layer of the atmosphere.

In this case it is necessary to take into account that the nature of the interaction process in three-phase system "dust-liquid-air" is influenced by hydrodynamic, aerodynamic, chemical and physical factors.

A number of methods for implementing the hydrodynamic method, characterized by the lowest liquid consumption, include a foam method for controlling dust, which achieves the highest value of dust retention efficiency (dust shielding). The efficiency of the dust retention process can be controlled studying and making purposeful changes in the properties of the foam $[3,4]$.
Foam, which is a coarsely dispersed colloidal system, is characterized by the following main parameters: density, dispersity, thermal conductivity, electrical conductivity, optical properties, and stability and multiplicity, which are the main parameters [5].

The foam density is characterized by the density of the foam solution and the air enclosed in the bubbles of the foam and depends on the ratio of the liquid and gaseous phases and can vary within half the value of the density of the liquid phase to value close to 0 .

The dispersion of the foam used for dust suppression is estimated by the average diameter of the bubbles, the values of which vary over a wide range - from 0.01 to $30 \mathrm{~mm}$.

The thermal conductivity of the foam is characterized by a corresponding coefficient and is determined by the formula:

$$
\lambda=0,67 \cdot\left(\alpha \cdot \lambda_{\mathrm{s}}\right)+(1-\alpha) \cdot \lambda_{\mathrm{a}}, W /(m \cdot K),
$$

where $\alpha$ is the degree of foam filling by the liquid phase; $\lambda_{\mathrm{s}}, \lambda_{\mathrm{a}}$ - respectively, the coefficients of thermal conductivity of the foam solution and air, $W /(m \cdot K)$.

The electrical conductivity of the foam is given by:

$$
\beta_{f}=2 \cdot \beta_{1} / 3 \cdot K_{f}, \mathrm{C} / \mathrm{m}^{3} \text {, }
$$

where $\beta_{\mathrm{f}}, \beta_{1}-$ respectively, the specific electrical conductivity of the foam and the intra-film liquid, $\mathrm{C} / \mathrm{m}^{3}$.

The optical properties of the foam are determined respectively by the coefficients of reflection, refraction, and absorption of the light flux by the sides (films) of the foam bubbles and depend on the thickness of the films themselves, as well as the foam layer.

\footnotetext{
Corresponding author: izos-rgsu@mail.ru
} 
In order to characterize the state of foam in practice, one of the main parameters uses the foam resistance $\tau$, defined by the formula:

$$
\tau_{n}=Q_{r} \cdot K_{f} / V_{f}, \mathrm{c},
$$

where $Q_{r}$ is the flow rate of the foamer solution, $\mathrm{m}^{3} / \mathrm{s}$; $V_{f}$ is the specified volume of foam, $\mathrm{m}^{3}$.

Persistence is characterized by the time of individual bubbles existence or a certain volume of foam and is determined mainly by the nature of the foaming agent, its concentration. Depending on the nature of the foaming agent, the stability of the foam can reach a maximum value at some other rates of concentration of the foaming agent, after which it decreases almost to zero, which is typical for low-molecular compounds.

The effects of Marangoni and Gibbs are among the factors that ensure the stability of the foam. The Marangoni effect improves resistance, manifesting itself the ability of thin films to react to local thickness changes. The Gibbs effect develops itself when under the influence of mechanical or thermal perturbations, causing the film to stretch, local surface tension differences arise and cause the flow of surfactant molecules in the adsorption layer toward a lower concentration-toward the stretched part of the film $[5,6]$.

Another basic parameter characterizing the state of the foam is its multiplicity $K_{n}$, which is in a certain dependence on the resistance and is the ratio of the volume $V_{n}$ of the foam to the volume $V_{p}$ of the liquid that went into its formation:

$$
K_{f}=V_{f} / V_{s}=0.78 \cdot D_{2} \cdot \pi \cdot g \cdot v_{\mathrm{s}} / Q_{r},
$$

where $D_{f g}$ is the diameter of the barrel of the foam generator, $\mathrm{m}$; $v_{\mathrm{c}}$ - speed of passage of foam through an output mouth of the foam generator, $\mathrm{m} / \mathrm{s}$.

In addition to the listed basic parameters for the characteristics of the foaming process, two additional criteria are additionally used:

- the first criterion of foaming (Dyakonov's criterion) $D_{1}$, characterizing the dependence of foam stability on the degree of dynamic external conditions:

$$
D_{1}=\tau_{d} / \tau_{s t},
$$

where $\tau_{d}, \tau_{s t}$-dynamic and static resistance of foam, s.

- the second criterion of foaming $D_{2}$, characterizing the physicochemical properties of foaming agents solutions and allowing to describe the foaming process in both dynamic and static conditions:

$$
D_{2}=K_{f} \cdot \tau_{s t}
$$

Thus, the efficiency of the foam method of air dedusting can be controlled by changing the parameters discussed above with the appropriate organization of foam technology and the supply of foam to the dust suppression zone to provide a shielding effect.

\section{Materials and Methods}

Mathematical modeling of the dust-holding process with a foam layer was performed by us taking into account the peculiarities of source dust shielding with a foam layer, the properties of foam bubbles, screened dust particles and the dynamic conditions of the physical mechanisms display of their interaction.

\section{Results}

As a result, taking into account the probabilistic realization of the successive interdependent physical mechanisms of dust particles interaction $[7,8]$ with a foam layer, the dust suppression efficiency is described by the following dependence:

$$
E_{e f-d r}=1-\left(1-E_{e f(1)-d r}\right) \cdot\left(1-E_{e f(2)-d r}\right) \cdot\left(1-E_{e f(3)-d r}\right),
$$

where $E_{e f(1)-d r}, E_{e f(2)-d r}, E_{e f(3)-d r}$ - respectively efficiencies caused by the action of inertia forces; forces of elastic interaction and electric forces.

The efficiency $E_{e f(1)-d r}$ depends on inertial sedimentation coefficient Kin and is determined by the formula:

$$
E_{e f(1)-d r}=1-K_{i n},
$$

The coefficient $K_{\text {in }}$ is determined [9]:

- under the condition that the velocity of the bubbles is less than the velocity at which the bubbles are fractionized, that is, $v \mathrm{~b}<\mathrm{vfr}$, according to the formula:

$$
K_{\text {in }}=\mathrm{Stk} /\left(\mathrm{Stk}+A_{1}\right) \text {, }
$$

- in case $v_{b} \geq v_{f r}$, then the process of bubbles fragmentation is of a mass character and $E_{e f(1)-d r}=0$.

The value of Stk conf depends on the foam volume shape, located in the form of the layer above the source of dust emission [10]. In addition, the configuration of the dust source itself also affects the Stk value.

The Stokes number Stk is determined by the formula:

$$
\mathrm{Stk}=d_{p} \cdot v \cdot \rho_{h} / 18 \cdot \mu_{i} \cdot l_{1},
$$

where $d$ is the particle diameter, $\mathrm{m} ; \mathrm{v}_{6 n}$ - speed of unperturbed air stream, running on an obstacle in the form of a foam layer, $\mathrm{m} / \mathrm{sec} ; \rho_{u}$ is the dust material density, $\mathrm{kg} / \mathrm{m}^{3} ; \mu_{v}$ is the coefficient of dynamic air viscosity equal to $1.82 \cdot 10^{-5} \mathrm{~Pa} \cdot \mathrm{s} ; l_{1}$ is the characteristic size of the streamlined obstacle in the form of a foam layer, $\mathrm{m}$, which for a foam layer (at $l_{n}=0$ ) becomes 1 .

\section{Discussion}

In the evaluationof the coefficient $A_{1}$ various discrepancies are observed in different authors' works $[11,12]$, that can be explained by the dependence of this coefficient on the particle sizes, the motion velocity, the bubbles velocity, and a number of other factors.

Regarding the foamy method for the implementation of the dust retention process, it is possible to extract that dust particles with a size dcr can not reach the foam layer, since their motion is so slowed that they lose their inertia and under the influence of the velocity components perpendicular to the direction of the main dust particle flow get demolished in parallel with the inner foam layer.

Taking into account the experimental data and the method presented in [13], the dependence of the 
coefficient $A_{1}$ on the ratio of the actual dimensions dp of dust particles and their critical size der was obtained for the foamed method:

$$
A_{1}=2 \cdot 10^{n 1},
$$

where $n_{1}$ is a coefficient that takes into account the ratio of the actual dimensions of dust particles $\mathrm{dp}$ and their critical size $d_{c r}$.

The value of $\mathrm{Stk}_{\mathrm{cr}}$. depends on the shape of the foam volume located in the form of a layer above the source of dust emission. In addition, the configuration of the dust source itself also affects the Stk value. Assuming inertial deposition of particles on the foam layer similar to deposition on a plate, we assume for the dust retention process:

- $\mathrm{Stk}_{\mathrm{cr}}=0,28$ on the source of dust emission of a rectangular section (which is typical for the conveyor belt surface);

- $\mathrm{Stk}_{\mathrm{cr}}=0,1$ on the source of dust emission of square or round section (which is a characteristic of the feed port or the delivery orifice of the overload nodes).

The process of shielding dust particles with a foam layer is characterized by the efficiency $E_{e f(2)}$-n which value depends mainly on the properties of the foam and the speed of the meeting of the dust particles with the foam bubbles. Taking into account the results of the experimental and theoretical studies presented in [14, $15]$ with respect to the foam layer the quantity $E_{e f(2)-d r}$ is determined by the formula:

$$
\left.E_{e f(2)-d r}=1-\exp \left[-0.693 \cdot\left(v_{c r}^{s} / v\right) 2\right) 2\right],
$$

where $v_{c r}^{s}$ is the critical velocity of a dust particle at which the foam bubble can collapse under a shielding (elastic) interaction, $\mathrm{m} / \mathrm{s}$.

The value of vscr can be determined on the basis of [16] taking into account the elastic properties of the bubble film:

$$
v_{c r}^{s}=\left[2 \pi \cdot \sigma \cdot d p / m_{p} \cdot\left[\delta / \delta_{c r} \cdot\left(1+d_{p} / d_{n} \cdot\left(d_{p}+\delta_{c r}\right)\right)-1\right],\right.
$$

where $\delta, \delta_{c r}$ - respectively, the average and critical thickness of the foam bubbles films, $\mathrm{m} ; m_{p}$ is the mass of a dust particle, $\mathrm{kg}$.

It is known that the thickness of the foam bubbles films varies with time due to evaporation, the outflow of the solution from the film, the entrainment of the solution by the air stream, etc., and depends on the film deflection wavelength, surface tension and other factors, the bubble does not collapse before and during the interference fringes emergence and is destroyed after their disappearance, that is, with the film thickness up to $7,6 \cdot 10^{-7} \mathrm{~m}$, and also up to $4 \cdot 10^{-7} \mathrm{~m}[17]$.

The mass of a dust particle $\mathrm{mp}$, the value of which depends on the density of its material and dimensions, can be determined by the formula:

$$
m=\pi \cdot d_{p}^{3} \rho_{p} / 6 .
$$

Capture of dust particles by foam under the action of electric forces $[17,18]$ is characterized by the efficiency $E_{e f(3)-d r}$.
Analysis of the experimental data $[19,20]$ made it possible to obtain the dependence of the efficiency of dust particles capture under the action of electric forces:

$$
E_{e f(3)-d r}=1,7 \cdot 10^{-3} \cdot q^{0,65},
$$

where $q_{0}$ is the specific electrical charge of foam, $\mathrm{C} / \mathrm{m}$.

It should be mentioned that with artificial foam electrification the specific charge $q_{0}$ depends on the method of foam recharging and the potential value.

With allowance for equations (8)-(15) the dependence $(7)$ of the efficiency of dust retention by a foam method using a foam layer takes the form:

$$
\begin{gathered}
E_{e f(3)-d r}=-d_{p} \cdot v \cdot \rho_{p} /\left(d_{p} \cdot v \cdot \rho_{p}+360 \cdot \mu \cdot 1\right) \cdot \exp \left[-7,48 \cdot \sigma / v^{2} \cdot \rho_{p} \cdot\right. \\
\left.\cdot\left(1 / d_{p}+2,1 \cdot d_{p} / d_{f}\left(d_{p}+4 \cdot 10^{-7}\right)\right)\right] \cdot\left(1,7 \cdot 10^{-3} \cdot q^{0,65}\right) .
\end{gathered}
$$

\section{Conclusions}

Thus, the performed mathematical modeling of the complex dynamic technical system "belt conveyor technology of dust retaining" provides the possibility of predictive calculation of the efficiency of dust holding with a foam layer, taking into account the peculiarities of its interaction with dust aerosol.

\section{References}

1. Bespalov V.I., Gurova O.S., Yudina N.V. Improvement of methods and means for dust removal of the air environment at concrete mixing departments of factories of reinforced concrete products and structures (Rostov State Civil Engineering University, Rostov-on-Don, 2015) [in Russian]

2. Bespalov V.I., Gurova O.S., Samarskaya N.S., Paramonova O.N. Industrial ecology (Rostov State Civil Engineering University, Rostov-on-Don, 2009) [in Russian]

3. Bespalov V.I., Gurova O.S., J. Scientific review, no.6, 193-196 (2012) [in Russian]

4. Dyakonov G.K. Questions of the theory of similarity in the field of physical and chemical processes (USSR Academy of Sciences, Moscow, 1956) [in Russian]

5. V.I. Bespalov, N.A. Strahova, Dedusting in the construction: intercollegiate collection, (RISI, Rostov-on-Don, 1990)

6. Bespalov V.I., Gurova O.S., Samarskaya N.S., Ingenerniy vestnik Dona, no. 4 (2013) [in Russian]

7. Ann T.W. Yu, Yuzhe Wu, Bibo Zheng, Xiaoling Zhang, Liyin Shen, Habitat International, no.44, 77185 (2014)

8. Tikhomirov V. K. The foam. Theory and practice of its obtaining and destruction, (Chemistry, Moscow, 1983) [in Russian]

9. Abramzon A. A. Surface-active substances. Properties and applications (Chemistry, Leningrad, 1981) [in Russian] 
10. Raist, P. Aerosols. Introduction to theory, (Mir, Moscow, 1987)

11. Bespalov V.I., Gurova O.S., Samarskaya N.S., Lysova E.P., Mishchenko A.N., Biosciences biotechnology research Asia, no.3, 1615-1620 (2014)

12. Bespalov V.I., Gurova O.S., Ingenerniy vestnik Dona, no. 3 (2013)

13. Daniela Vallero. Fundamentals of Air Pollution fourth edition, (Civil and Environmental Engineering Department Pratt School of Engineering Duke University, Durham, 2007)

14. V. I. Bespalov, O. S. Gurova, N. S. Samarskaya and others, J. Biosciences Biotechnology Research Asia, no.2, 1587-1596 (2015)

15. Romaniuk T.F., Technology of building materials production, (Federal Agency for Education, Tomsk State University. Architectural and Construction Universit, Tomsk, 2006)

16. Bespalov V.I., Gurova O.S., Meshcheryakov S.V., Processes and apparatus for reducing air pollution (Rostov State Civil Engineering University, Rostov-on-Don, 2014)

17. V.I. Bespalov, O. S. Gurova, Int. Scient. Pract. Conf., 35 (2015).

18. V. I. Bespalov, O. S. Gurova, E. P. Lysova, J. Biosciences Biotechnology Research Asia, no.2, 1459-1470 (2015)

19. Gurova O. S., Bespalov V.I., Paramonova O.N., J. Naukovedenie, no. 6, (2015)

20. Gurova O. S., Bespalov V.I., Paramonova O.N. J. Naukovedenie, no. 6 (2015) 